\title{
Biologia e exigências térmicas do ácaro-vermelho (Tetranychus ludeni Zacher) em folhas de algodoeiro ${ }^{(1)}$
}

\author{
Carlos Alberto Domingues da Silva(2)
}

\begin{abstract}
Resumo - O objetivo deste trabalho foi estudar a biologia e as exigências térmicas de Tetranychus ludeni Zacher (Acari: Tetranychidae) em folhas de algodoeiro (Gossypium hirsutum L. r. latifolium (Hutch)). Foram utilizadas câmaras climatizadas, ajustadas nas temperaturas de $20,23,25,28$ e $30^{\circ} \mathrm{C}$, umidade relativa de $70 \%$ e fotófase de 12 horas. Os períodos de desenvolvimento de ovo a adulto variaram de $20,77\left(20^{\circ} \mathrm{C}\right)$ a 8,50 dias $\left(30^{\circ} \mathrm{C}\right)$, em fêmeas, e de $18,83\left(20^{\circ} \mathrm{C}\right)$ a 7,75 dias $\left(30^{\circ} \mathrm{C}\right)$, em machos. As temperaturas-base, estimadas pelo método da intersecção de $\mathrm{X}$, para os períodos de desenvolvimento de ovo-adulto, foram de $14,05^{\circ} \mathrm{C}$ para fêmeas e $13,91^{\circ} \mathrm{C}$ para machos, enquanto os valores da constante térmica estimados de acordo com a lei de Réamur foram de 138,34 graus-dia, para fêmeas e 130,91 graus-dia, para machos. Na temperatura de $30^{\circ} \mathrm{C}$ foram observados os maiores valores de razão intrínseca de crescimento $(0,418)$, número de ovos de fêmeas/dia $(3,47)$, fecundidade $(61,29)$ e taxa líquida de reprodução $(48,00)$ e o menor valor para o tempo médio de uma geração $(9,27)$.
\end{abstract}

Termos para indexação: Gossypium hirsutum, termorregulação, temperatura.

\section{Biology and thermal requirement of Tetranychus ludeni Zacher on cotton leaves}

\begin{abstract}
The objective of this work was to study the biology and thermal requirement of Tetranychus ludeni Zacher (Acari: Tetranychidae) on leaves of cotton (Gossypium hirsutum L. r. latifolium (Hutch)). Bioclimatic chambers at $20,23,25,28$ and $30^{\circ} \mathrm{C}$, relative humidity of $70 \pm 10 \%$, and a 12 hours:12 hours (L:D) photoperiod were utilized. Development period of egg-adult, varied from $20.77\left(20^{\circ} \mathrm{C}\right)$ to 8.50 days $\left(30^{\circ} \mathrm{C}\right)$, for males and from $18.83\left(20^{\circ} \mathrm{C}\right)$ to 7.75 days $\left(30^{\circ} \mathrm{C}\right)$, for females. The threshold temperature of development, estimated by $\mathrm{X}$ intercession method for development period of egg-adult were $14.05^{\circ} \mathrm{C}$, for females and $13.91^{\circ} \mathrm{C}$, for males. While those values of thermal constant estimated by Réamur law were 138.34 day-degrees for females and 130.91 day-degrees for males. At $30^{\circ} \mathrm{C}$ of temperature, the highest values of the intrinsic rate of increase $(0.418)$, number of eggs/females/day (3.47), fecundity (61.29), reproductive rate net (48.00), and minimum value to the mean generation time (9.27) were shown.
\end{abstract}

Index terms: Gossypium hirsutum, thermoregulation, temperature.

\section{Introdução}

Diversas espécies de ácaros podem causar significativas reduções na colheita e na qualidade da fibra do algodão e, também, afetar outros atributos, como a viabilidade da semente (Wilson, 1993). Entre essas espécies, o ácaro-vermelho, Tetranychus ludeni, encontra-se amplamente distribuído nos trópicos, atravessando o sul dos Estados Unidos, Mé-

\footnotetext{
(1) Aceito para publicação em 17 de julho de 2001

(2) Embrapa-Centro Nacional de Pesquisa de Algodão, Caixa Postal 174, CEP 58107-720 Campina Grande, PB E-mail: carlos@cnpa.embrapa.br
}

xico, Américas Central e do Sul, África do Sul e Austrália (Jeppson et al., 1975). Estudos sobre sua biologia têm sido realizados sobre a erva aquática, Eichhornia crassipes (Ansari \& Pawar, 1992), o feijão-preto Phaseolus vulgaris L. (Morros \& Aponte, 1994) e sobre sua preferência varietal em variedades de Solanum melongena L. (Reddy \& Baskaran, 1991) além de trabalhos sobre sua bioecologia (Kumar \& Sharma, 1993; Sumangala \& Haq, 1994; Singh, 1995).

No Brasil, infestações desse ácaro têm sido observadas em lavouras de algodão no Paraná (Bleicher, 1993). O ataque tem início geralmente nas folhas mais velhas (baixeiro) para depois generalizar-se por toda a planta, inclusive nas folhas do ponteiro (Calcagnolo 
\& Sauer, 1955; Calcagnolo, 1963; Nakano et al., 1981). As folhas atacadas apresentam, de início, pequenas manchas avermelhadas entre as nervuras das folhas, as quais coalescem, tomando toda a folha que, posteriormente, seca e cai.

Apesar de essa espécie ser bastante estudada em diversas partes do mundo, constata-se que poucos são os estudos encontrados na literatura brasileira sobre sua biologia e exigências térmicas. A temperatura, segundo Jeppson et al. (1975), é o mais importante fator ambiental que afeta a população de Tetraniquídeos. O seu efeito sobre a taxa de desenvolvimento dos ácaros tem demonstrado que altas temperaturas promovem decréscimo na longevidade e aumento na taxa de oviposição e fecundidade em diversas espécies (Nickel, 1960). Os limites da temperatura a partir dos quais os ácaros deixam de sobreviver variam muito com as espécies e com a sua fase de desenvolvimento (Kim et al., 1993; Popov, 1994; Alvarez et al., 1997; Bonato, 1999). Além disso, tanto para as plantas como para os insetos e ácaros que delas dependem, os respectivos ritmos de desenvolvimento, mais do que com os valores da temperatura verificados ao longo do tempo, se correlacionam com o número de graus de temperatura que se somam durante determinado período, ou seja, com o somatório de unidades térmicas denominadas graus-dia (Carvalho, 1986). Este somatório de unidades, considerado apenas com relação a valores da temperatura acima do zero de desenvolvimento, constitui-se num fator de fundamental importância para se determinar os efeitos da temperatura sobre os insetos e ácaros e sobre o próprio condicionalismo do ambiente, notadamente sobre o sincronismo que se estabelece entre as diferentes fases de desenvolvimento desses artrópodos-praga e seus hospedeiros. Sabe-se que a empírica relação entre a temperatura e a taxa de desenvolvimento embrionário e pós-embrionário dos ácaros tetraniquídeos é curvilínea. Então, pode-se estimar os valores de temperatura-base e constante térmica desses ácaros, através da linearização da curva obtida em laboratório.

O objetivo deste trabalho foi obter informações sobre a biologia de Tetranychus ludeni e as exigências térmicas para as diferentes fases de seu ciclo biológico em folhas de algodoeiro.

\section{Material e Métodos}

O trabalho foi realizado no laboratório de Entomologia da Embrapa-Centro Nacional de Pesquisa de Algodão, sob condições controladas nas temperaturas de 20,23, 25, 28 e $30^{\circ} \mathrm{C}, 70 \pm 10 \%$ de umidade relativa e 12 horas de fotófase.

Na obtenção dos ovos, duzentas fêmeas de T. ludeni foram obtidas da criação-estoque do referido laboratório, e transferidas isoladamente para unidades de criação, para efetuar postura. A espécie foi identificada com auxílio de microscópio óptico, através da montagem do macho e análise morfológica do edeago de acordo com Pritchard \& Baker (1955).

Cada unidade de criação foi constituída por um disco de $2,5 \mathrm{~cm}$ de diâmetro, tomado da face ventral de uma folha de algodão cultivar CNPA ITA 90. Essas unidades de criação foram colocadas sobre uma espuma de poliuretano previamente saturada com água destilada, medindo1,5 cm de espessura e recoberta por papel de filtro dentro de uma bandeja plástica de $20 \mathrm{~cm}$ de diâmetro e $3 \mathrm{~cm}$ de altura. A espuma foi umedecida diariamente, para manter os discos de folhas de algodão túrgidos e evitar a fuga dos ácaros. Doze horas após a transferência, as fêmeas foram eliminadas, e os ovos, individualizados em 50 unidades de criação, para cada temperatura testada

As observações biológicas foram efetuadas diariamente, sempre às $8 \mathrm{~h}$ e às $16 \mathrm{~h}$, com o auxílio de um estereomicroscópio.

Em cada temperatura foram quantificadas as durações e sobrevivência das fases de ovo, larva ativa e quiescente, protoninfa ativa e quiescente, deutoninfa ativa e quiescente e período de ovo à emergência de adultos machos e fêmeas $\mathrm{Na}$ fase adulta, observaram-se períodos de pré-oviposição, oviposição, pós-oviposição, longevidade e fecundidade. As diferenças nas durações de desenvolvimento entre machos e fêmeas de T. ludeni foram comparadas pelo teste $\mathrm{t}$, enquanto os valores médios obtidos nos períodos de pré-oviposição, oviposição, pós-oviposição, longevidade e taxas de fecundidade e número de ovos/fêmea/dia obtidos em cada temperatura foram comparados pelo teste de Tukey a 5\% de probabilidade. Na determinação da fecundidade e da razão sexual da progênie em cada temperatura, adultos machos recém-emergidos foram transferidos isoladamente para novas arenas contendo uma fêmea recém-emergida. O número de ovos depositados nessas arenas foi quantificado diariamente, até as $16 \mathrm{~h}$, e os dados obtidos, utilizados para a construção da Tabela de vida de fertilidade, de acordo com Birch (1948). As exigências térmicas $(\mathrm{K})$ e temperaturas-base $(\mathrm{Tb})$ foram estimadas para cada uma das fases imaturas e de ovo à emergência de adultos machos e fêmeas, de acordo com Lei de Réamur e com o método da intersecção de $\mathrm{X}$, respectivamente. 


\section{Resultados e Discussão}

As maiores perdas foram registradas na fase de ovo de T. ludeni, cujas viabilidades variaram de 56 $\left(20^{\circ} \mathrm{C}\right)$ a $80 \%\left(28^{\circ} \mathrm{C}\right)$ (Tabela 1). Desta forma, exceto a $20^{\circ} \mathrm{C}$, as viabilidades dos ovos foram superiores a $70 \%$, demonstrando que temperaturas situadas den- tro dos limites de 23 a $30^{\circ} \mathrm{C}$ são mais favoráveis ao desenvolvimento dos ovos de T. ludeni. Esses resultados são condizentes com a faixa ótima de temperatura $\left(24 \mathrm{a} 29^{\circ} \mathrm{C}\right)$ de desenvolvimento evidenciada por Boudreaux (1963), para diversas espécies de tetraniquídeos.

Tabela 1. Sobrevivência e duração média de desenvolvimento de cada uma das fases imaturas de Tetranychus ludeni, às temperaturas de 20 a $30^{\circ} \mathrm{C}$, umidade relativa do ar de $70 \pm 10 \%$ e fotófase de 12 horas. Campina Grande, 1999.

\begin{tabular}{|c|c|c|c|c|c|c|c|}
\hline \multirow[t]{2}{*}{ Fase } & \multirow{2}{*}{$\begin{array}{c}\text { Temperatura } \\
\left({ }^{\circ} \mathrm{C}\right)\end{array}$} & \multirow{2}{*}{$\begin{array}{l}\text { Número de } \\
\text { indivíduos }\end{array}$} & \multirow{2}{*}{$\begin{array}{c}\text { Sobrevivência } \\
(\%)\end{array}$} & \multicolumn{4}{|c|}{ Duração (dia) \pm EP } \\
\hline & & & & Fêmea & $\mathrm{N}^{(1)}$ & Macho & $\mathbf{N}^{(1)}$ \\
\hline \multirow[t]{5}{*}{ Ovo } & 20 & 28 & 56,00 & $7,95 \pm 0,18$ & 11 & $7,50 \pm 0,34^{\mathrm{ns}}$ & 3 \\
\hline & 23 & 36 & 72,00 & $6,70 \pm 0,21$ & 22 & $6,55 \pm 0,14^{\mathrm{ns}}$ & 10 \\
\hline & 25 & 37 & 74,00 & $5,71 \pm 0,15$ & 28 & $5,36 \pm 0,07^{*}$ & 7 \\
\hline & 28 & 40 & 80,00 & $4,25 \pm 0,20$ & 33 & $4,08 \pm 0,07^{\mathrm{ns}}$ & 6 \\
\hline & 30 & 36 & 72,00 & $3,50 \pm 0,45$ & 28 & $3,57 \pm 0,12^{\mathrm{ns}}$ & 2 \\
\hline \multirow[t]{5}{*}{ Larva } & 20 & 21 & 75,00 & $3,50 \pm 0,32$ & 11 & $3,32 \pm 0,17^{\mathrm{ns}}$ & 3 \\
\hline & 23 & 35 & 97,22 & $1,73 \pm 0,11$ & 22 & $1,40 \pm 0,17^{\mathrm{ns}}$ & 10 \\
\hline & 25 & 37 & 100,00 & $1,64 \pm 0,07$ & 28 & $1,43 \pm 0,14^{\mathrm{ns}}$ & 7 \\
\hline & 28 & 40 & 100,00 & $1,25 \pm 0,14$ & 33 & $1,05 \pm 0,05^{\mathrm{ns}}$ & 6 \\
\hline & 30 & 34 & 94,44 & $0,86 \pm 0,05$ & 28 & $1,00 \pm 0,19^{\mathrm{ns}}$ & 2 \\
\hline \multirow[t]{5}{*}{ Larva quiescente } & 20 & 15 & 71,43 & $2,18 \pm 0,18$ & 11 & $1,50 \pm 0,34^{\mathrm{ns}}$ & 3 \\
\hline & 23 & 34 & 97,14 & $2,05 \pm 0,07$ & 22 & $1,80 \pm 0,11^{\mathrm{ns}}$ & 10 \\
\hline & 25 & 37 & 100,00 & $1,00 \pm 0,07$ & 28 & $1,07 \pm 0,14^{\mathrm{ns}}$ & 7 \\
\hline & 28 & 40 & 100,00 & $0,94 \pm 0,05$ & 33 & $1,00 \pm 0,13^{\mathrm{ns}}$ & 6 \\
\hline & 30 & 33 & 97,06 & $0,71 \pm 0,06$ & 28 & $1,00 \pm 0,22^{\mathrm{ns}}$ & 2 \\
\hline \multirow[t]{5}{*}{ Protoninfa } & 20 & 15 & 100,00 & $1,95 \pm 0,07$ & 11 & $1,50 \pm 0,14^{*}$ & 3 \\
\hline & 23 & 34 & 100,00 & $1,34 \pm 0,09$ & 22 & $1,05 \pm 0,13^{\mathrm{ns}}$ & 10 \\
\hline & 25 & 36 & 97,30 & $1,09 \pm 0,06$ & 28 & $1,07 \pm 0,13^{\mathrm{ns}}$ & 7 \\
\hline & 28 & 40 & 100,00 & $0,92 \pm 0,04$ & 33 & $0,67 \pm 0,12^{*}$ & 6 \\
\hline & 30 & 31 & 93,94 & $0,95 \pm 0,05$ & 28 & $0,50 \pm 0,17^{*}$ & 2 \\
\hline \multirow[t]{5}{*}{ Prot. quiescente } & 20 & 15 & 100,00 & $1,77 \pm 0,11$ & 11 & $1,50 \pm 0,22^{\mathrm{ns}}$ & 3 \\
\hline & 23 & 34 & 100,00 & $1,75 \pm 0,11$ & 22 & $1,55 \pm 0,16^{\mathrm{ns}}$ & 10 \\
\hline & 25 & 36 & 100,00 & $1,73 \pm 0,08$ & 28 & $1,07 \pm 0,17^{*}$ & 7 \\
\hline & 28 & 40 & 100,00 & $0,66 \pm 0,04$ & 33 & $0,58 \pm 0,10^{\mathrm{ns}}$ & 6 \\
\hline & 30 & 31 & 100,00 & $0,59 \pm 0,04$ & 28 & $0,50 \pm 0,17^{\mathrm{ns}}$ & 2 \\
\hline \multirow[t]{5}{*}{ Deutoninfa } & 20 & 15 & 93,33 & $2,00 \pm 0,33$ & 11 & $1,83 \pm 0,16^{\mathrm{ns}}$ & 3 \\
\hline & 23 & 33 & 97,06 & $1,61 \pm 0,15$ & 22 & $1,25 \pm 0,22^{\mathrm{ns}}$ & 10 \\
\hline & 25 & 35 & 100,00 & $1,00 \pm 0,07$ & 28 & $1,07 \pm 0,16^{\mathrm{ns}}$ & 7 \\
\hline & 28 & 39 & 97,50 & $1,16 \pm 0,05$ & 33 & $1,00 \pm 0,14^{\mathrm{ns}}$ & 6 \\
\hline & 30 & 31 & 100,00 & $1,03 \pm 0,06$ & 28 & $0,75 \pm 0,22^{\mathrm{ns}}$ & 2 \\
\hline \multirow[t]{5}{*}{ Deut. quiescente } & 20 & 15 & 100,00 & $1,73 \pm 0,10$ & 11 & $1,67 \pm 0,19^{\mathrm{ns}}$ & 3 \\
\hline & 23 & 32 & 96,97 & $1,73 \pm 0,13$ & 22 & $1,70 \pm 0,19^{\mathrm{ns}}$ & 10 \\
\hline & 25 & 35 & 100,00 & $1,46 \pm 0,07$ & 28 & $1,21 \pm 0,14^{\mathrm{ns}}$ & 7 \\
\hline & 28 & 39 & 100,00 & $0,98 \pm 0,03$ & 33 & $0,75 \pm 0,08^{\mathrm{ns}}$ & 6 \\
\hline & 30 & 30 & 96,77 & $0,79 \pm 0,05$ & 28 & $0,50 \pm 0,18^{\mathrm{ns}}$ & 2 \\
\hline \multirow[t]{5}{*}{ Ovo-adulto } & 20 & 15 & 28,00 & $20,77 \pm 0,27$ & 11 & $18,83 \pm 0,51^{*}$ & 3 \\
\hline & 23 & 32 & 64,00 & $16,75 \pm 0,23$ & 22 & $15,45 \pm 0,34^{*}$ & 10 \\
\hline & 25 & 35 & 70,00 & $13,29 \pm 0,09$ & 28 & $12,64 \pm 0,18^{*}$ & 7 \\
\hline & 28 & 39 & 78,00 & $9,79 \pm 0,09$ & 33 & $9,50 \pm 0,25^{\mathrm{ns}}$ & 6 \\
\hline & 30 & 30 & 60,00 & $8,50 \pm 0,12$ & 28 & $7,75 \pm 0,44^{\mathrm{ns}}$ & 2 \\
\hline
\end{tabular}

(1)Número de indivíduos que atingiram o estágio adulto. ${ }^{\text {ns Não-significativo. * }}$ Significativo a $5 \%$ de probabilidade pelo teste t. 
A temperatura afetou a duração do período de incubação dos ovos e independente da temperatura, o período de incubação foi sempre maior em relação aos demais estágios imaturos (Tabela 1).

Com exceção da temperatura de $20^{\circ} \mathrm{C}$, a sobrevivência de T. ludeni durante cada fase foi alta, sendo $28^{\circ} \mathrm{C}$ a temperatura que proporcionou as mais altas sobrevivências. Portanto, pode-se afirmar que temperatura constante ou inferior a $20^{\circ} \mathrm{C}$ desfavorece a sobrevivência de T. ludeni. Entre as fases imaturas, exceto a fase de ovo, as maiores perdas ocorreram na fase larval (Tabela 1).

A $25^{\circ} \mathrm{C}$ a duração das fases imaturas combinadas foi de 5,43 (incubação), 2,61 (larval), 2,69 (protoninfal) e 2,42 dias (deutoninfal). Esses resultados foram superiores aos valores de 4,68 (incubação), 1,75 (larval), 1,31 (protoninfal) e 1,85 dias (deutoninfal) obtidos por Morros \& Aponte (1994) em feijoeiro a $26^{\circ} \mathrm{C}$, sendo, porém, semelhantes aos valores de 4,00 (incubação), 3,00 (larval), 2,70 (protoninfal) e 2,80 dias (deutoninfal) constatados por Nakano et al. (1981) em algodoeiro. A duração do período de ovo-adulto para indivíduos que originaram fêmeas e machos, respectivamente, de 13,29 e 12,64 dias, foi superior aos valores de 9,98 e 9,25 dias, obtidos por Morros \& Aponte (1994) em feijoeiro, à temperatura de $26^{\circ} \mathrm{C}$, sendo, porém, semelhante aos 14 dias obtidos por Nakano et al. (1981) em algodoeiro.

A duração de desenvolvimento de T. ludeni variou em cada fase e entre os sexos, conforme a temperatura a que o ácaro foi submetido (Tabela 1). As fases imaturas de T. ludeni que originaram fêmeas apresentaram duração de desenvolvimento superior àquele que originou machos; entretanto, somente foram verificadas diferenças significativas (teste $t$, $\mathrm{P}=0,05)$ nas fases de ovo $\left(25^{\circ} \mathrm{C}\right)$, protoninfa $(20,28$ e $\left.30^{\circ} \mathrm{C}\right)$ e protoninfa quiescente $\left(25^{\circ} \mathrm{C}\right)$. A duração das fases imaturos, exceto a fase de ovo, foi semelhante dentro de cada uma das cinco temperaturas estudadas, variando de $0,44\left(30^{\circ} \mathrm{C}\right)$ a $1,77 \operatorname{dias}\left(20^{\circ} \mathrm{C}\right)$ para indivíduos que originaram fêmeas e de $0,50\left(30^{\circ} \mathrm{C}\right)$ a 1,82 dias $\left(20^{\circ} \mathrm{C}\right)$ para indivíduos que originaram machos.

A duração relativa das fases imaturas de T. ludeni foi semelhante; assim, os períodos de incubação, larva ativa, larva quiescente, protoninfa ativa, protoninfa quiescente, deutoninfa ativa e deutoninfa quiescente, representaram, respectivamente, cerca de $38-46,10-17,8-13,6-11,6-8,10-12$ e $6-9 \%$ do total dos períodos de ovo-adulto tanto para indivíduos que originaram fêmeas quanto para aqueles que originaram machos.

Nas menores temperaturas $\left(20\right.$ e $\left.23^{\circ} \mathrm{C}\right)$, as fêmeas de T. ludeni necessitam aproximadamente de dois dias após a cópula, para iniciar a postura, enquanto nas temperaturas maiores $\left(28^{\circ} \mathrm{C}\right.$ e $\left.30^{\circ} \mathrm{C}\right)$ necessitam somente de um dia (Tabela 2). Os períodos de pré-oviposição e oviposição foram diminuindo, à medida que se aumentavam as temperaturas. O período de pós-oviposição foi semelhante nas cinco temperaturas estudadas.

A temperatura afetou a longevidade de T. ludeni, com tendência de redução na duração à medida que se aumentavam as temperaturas, e, independentemente da temperatura, a longevidade das fêmeas foi sempre maior que a dos machos (Tabela 3).

Os períodos de duração de desenvolvimento de ovo, larva ativa e quiescente, protoninfa ativa e

Tabela 2. Duração (dias) dos períodos de pré-oviposicão, oviposição e pós-oviposicão de Tetranychus ludeni, às temperaturas de 20 a $30^{\circ} \mathrm{C}$, umidade relativa do ar $70 \pm 10 \%$ e fotófase de 12 horas ${ }^{(1)}$.

\begin{tabular}{ccccc}
\hline Temperatura $\left({ }^{\circ} \mathrm{C}\right)$ & Número de fêmeas & Pré-oviposicão & Oviposição & Pós-oviposição \\
\hline 20 & 11 & $1,88 \pm 0,43 \mathrm{a}$ & $22,22 \pm 11,01 \mathrm{a}$ & $1,25 \pm 0,87 \mathrm{a}$ \\
23 & 22 & $1,70 \pm 0,62 \mathrm{a}$ & $20,98 \pm 11,02 \mathrm{a}$ & $1,23 \pm 0,91 \mathrm{a}$ \\
25 & 28 & $1,58 \pm 0,74 \mathrm{a}$ & $14,13 \pm 04,90 \mathrm{~b}$ & $1,38 \pm 0,44 \mathrm{a}$ \\
28 & 33 & $1,03 \pm 0,38 \mathrm{~b}$ & $11,77 \pm 05,11 \mathrm{c}$ & $1,08 \pm 1,47 \mathrm{a}$ \\
30 & 28 & $1,00 \pm 0,00 \mathrm{~b}$ & $10,00 \pm 00,65 \mathrm{c}$ & $0,71 \pm 0,75 \mathrm{a}$ \\
\hline CV $(\%)$ & - & 19,14 & 6,76 & 15,35 \\
\hline
\end{tabular}

${ }^{(1)}$ Médias seguidas pela mesma letra não diferem entre si, pelo teste de Tukey a $5 \%$ de probabilidade. 
quiescente, deutoninfa ativa e quiescente, de ovo à emergência de adultos que originaram fêmeas e machos de T. ludeni, apresentaram relação linear positiva entre a velocidade de desenvolvimento e a temperatura, entre os limites de 20 e $30^{\circ} \mathrm{C}$ (Tabela 4). Dentro deste limite, foram observadas diferenças de velocidades de desenvolvimento entre fases imaturas e entre os sexos. Desta forma, ácaros cuja fase larval (ativo e quiescente) originou fêmeas apresen-

Tabela 3. Médias ( \pm erro padrão) de longevidade de fêmeas e machos de Tetranychus ludeni, às temperaturas de 20 a $30^{\circ} \mathrm{C}$, umidade relativa do ar de $70 \pm 10 \%$ e fotófase de 12 $\operatorname{horas}^{(1)}$.

\begin{tabular}{clr}
\hline Temperatura $\left({ }^{\circ} \mathrm{C}\right)$ & \multicolumn{1}{c}{ Fêmea } & \multicolumn{1}{c}{ Macho } \\
\hline 20 & $24,16 \pm 9,33 \mathrm{a}$ & $16,94 \pm 5,62 \mathrm{a}$ \\
23 & $23,74 \pm 10,94 \mathrm{a}$ & $14,50 \pm 0,58 \mathrm{a}$ \\
25 & $17,38 \pm 4,84 \mathrm{ab}$ & $8,83 \pm 2,63 \mathrm{~b}$ \\
28 & $13,88 \pm 4,29 \mathrm{~b}$ & $7,75 \pm 2,22 \mathrm{~b}$ \\
30 & $11,93 \pm 0,53 \mathrm{~b}$ & $5,73 \pm 1,25 \mathrm{~b}$ \\
\hline
\end{tabular}

${ }^{(1)}$ Médias seguidas pela mesma letra na vertical não diferem entre si, pelo teste de Tukey a $5 \%$ de probabilidade. taram maior velocidade de desenvolvimento que aqueles que originaram machos; por outro lado, ácaros cujas fases de protoninfa ativa, deutoninfa ativa e quiescente originaram machos, apresentaram maior velocidade de desenvolvimento do que os que originaram fêmeas, conforme pode ser verificado pelos maiores coeficientes angulares das equações de regressão estimados com relação à esses estágios. No desenvolvimento de ovo-adulto que originou fêmeas e machos de T. ludeni, foram requeridos 138,34 e 130,91 graus-dia, respectivamente, acima dos limites inferiores de 14,05 e $13,91{ }^{\circ} \mathrm{C}$. As temperaturas-base para o período ovo-adulto em ácaros que originaram fêmeas e machos foram superiores às temperaturas-base de $10,30^{\circ} \mathrm{Ce} 13,20^{\circ} \mathrm{C}$, obtidas para T. evansi por Bonato (1999) e Moraes \& MacMurtry (1987), respectivamente. Os valores das constantes térmicas de graus-dia foram inferiores aos 148 graus-dia obtidos para T. evansi (Moraes \& MacMurtry, 1987). O tempo de desenvolvimento dos estágios imaturos combinados de T. ludeni decresceu rapidamente de 20 para $30^{\circ} \mathrm{C}$; esse decréscimo foi menor com o aumento da temperatura.

Tabela 4. Temperatura-base ( $\mathrm{Tb}$ ), constante térmica (K), intercepta (a) e coeficiente angular (b) da equação de regressão da velocidade de desenvolvimento das fases imaturas de fêmeas e machos de Tetranychus ludeni em algodoeiro, às temperaturas de 20 a $30^{\circ} \mathrm{C}$, umidade relativa do ar de $70 \pm 10 \%$ e fotófase de 12 horas $^{(1)}$.

\begin{tabular}{lrrrrrr}
\hline Fase & $\mathrm{Tb}\left({ }^{\circ} \mathrm{C}\right)$ & $\mathrm{K}$ (graus-dia) & \multicolumn{1}{c}{$\mathrm{a} \pm$ erro padrão } & $\mathrm{b} \pm$ erro padrão & $\mathrm{p}$ & $\mathrm{R}^{2}$ \\
\hline & \multicolumn{7}{c}{ Fêmea } \\
Ovo & 13,16 & 62,01 & $-0,212166 \pm 0,052264^{* *}$ & $0,016127 \pm 0,002054$ & 0,004 & 0,95 \\
Larva & 16,47 & 12,71 & $-1,295858 \pm 0,316272^{* *}$ & $0,078695 \pm 0,012428$ & 0,008 & 0,93 \\
Larva quiescente & 16,07 & 10,33 & $-1,556304 \pm 0,490138^{*}$ & $0,096828 \pm 0,019260$ & 0,015 & 0,89 \\
Protoninfa & 10,16 & 17,42 & $-0,583036 \pm 0,252062^{*}$ & $0,057391 \pm 0,009905$ & 0,010 & 0,92 \\
Prot. quiescente & 17,53 & 7,78 & $-2,252342 \pm 0,948577^{*}$ & $0,128462 \pm 0,037275$ & 0,041 & 0,80 \\
Deutoninfa & 8,12 & 21,60 & $-0,375903 \pm 0,454191^{\text {ns }}$ & $0,046298 \pm 0,017848$ & 0,081 & 0,69 \\
Deut. quiescente & 13,73 & 13,90 & $-0,987770 \pm 0,410322^{*}$ & $0,071953 \pm 0,016124$ & 0,021 & 0,87 \\
Ovo-adulto & 14,05 & 138,34 & $-0,101585 \pm 0,015930^{* *}$ & $0,007229 \pm 0,000626$ & 0,001 & 0,98 \\
\hline & \multicolumn{7}{c}{ Macho $^{*}$} & & \\
Ovo & 12,20 & 65,13 & $-0,187387 \pm 0,041832^{* *}$ & $0,015355 \pm 0,001643$ & 0,003 & 0,97 \\
Larva & 14,20 & 14,99 & $-0,947547 \pm 0,309746^{*}$ & $0,066706 \pm 0,012172$ & 0,012 & 0,91 \\
Larva quiescente & 6,03 & 23,05 & $-0,261788 \pm 0,425020^{\text {ns }}$ & $0,043379 \pm 0,016702$ & 0,081 & 0,69 \\
Protoninfa & 15,74 & 7,82 & $-2,013110 \pm 0,627416^{*}$ & $0,127871 \pm 0,024655$ & 0,014 & 0,90 \\
Prot. quiescente & 17,18 & 6,71 & $-2,559667 \pm 0,794901^{*}$ & $0,148959 \pm 0,031236$ & 0,018 & 0,88 \\
Deutoninfa & 12,06 & 14,24 & $-0,847369 \pm 0,271569^{* *}$ & $0,070248 \pm 0,010672$ & 0,007 & 0,94 \\
Deut. quiescente & 17,53 & 7,17 & $-2,445858 \pm 0,880815^{* *}$ & $0,139493 \pm 0,034612$ & 0,027 & 0,84 \\
Ovo-adulto & 13,91 & 130,91 & $-0,106251 \pm 0,021941^{* *}$ & $0,007639 \pm 0,000862$ & 0,003 & 0,96 \\
\hline
\end{tabular}

${ }^{(1)} \mathrm{D}=\mathrm{a}+\mathrm{bt}$, onde $\mathrm{D}$ : duração (em dias) e t: temperatura $\left({ }^{\circ} \mathrm{C}\right.$ ); $\mathrm{p}$ : nível de probabilidade (teste $\mathrm{t}$ ) referente a intercepta; $\mathrm{R}^{2}$ : coeficiente de determinação.

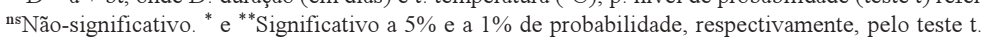


A razão intrínseca de crescimento $\left(\mathrm{r}_{\mathrm{m}}\right)$ de cada uma das cinco temperaturas estudadas foi calculada considerando-se a variação na razão sexual da progênie durante o período de oviposição (Tabela 5). Assim, os valores de $\mathrm{r}_{\mathrm{m}} \mathrm{e} \lambda$ (razão finita de aumento) a $20^{\circ} \mathrm{C}$ foram, respectivamente, de 0,152 e 1,164 fêmea/fêmea/dia, porém quando submetidos a temperatura de $30^{\circ} \mathrm{C}$, esses valores foram de $0,418 \mathrm{e}$ 1,519 fêmea/fêmea/dia, respectivamente. Por outro lado, houve tendência de redução dos valores obtidos para o tempo médio de uma geração com o aumento da temperatura, os quais variaram de 24,20 dias a $20^{\circ} \mathrm{C}$, até 9,27 dias a $30^{\circ} \mathrm{C}$, ou seja, pode-se obter 15,08 e 39,37 gerações de T. ludeni durante o ano, respectivamente, nas temperaturas de 20 e $30^{\circ} \mathrm{C}$. Os valores obtidos para a razão de crescimento $\left(R_{0}\right)$ variaram de 39,75 a $20^{\circ} \mathrm{C}$, até 48,00 a $30^{\circ} \mathrm{C}$; portanto, verifica-se tendência de aumento da taxa líquida de reprodução com o aumento da temperatura; havendo, também, tendência de aumento na fecundidade. À temperatura de $25^{\circ} \mathrm{C}$, os resultados de $46,86,0,222$ e 1,249 obtidos, respectivamente, para a razão de crescimento $\left(\mathrm{R}_{o}\right)$, a razão intrínseca de crescimento $\left(\mathrm{r}_{\mathrm{m}}\right) \mathrm{e}$ a razão finita de aumento $(\lambda)$ foram semelhantes aos valores de 44,6 $\left(\mathrm{R}_{\mathrm{o}}\right), 0,207$ fêmea/fêmea/dia $\left(\mathrm{r}_{\mathrm{m}}\right)$, 1,229 fêmea/fêmea/dia $(\lambda)$ e 46,8 $\left(R_{0}\right), 0,203$ fềmea/fê- mea/dia $\left(r_{m}\right)$ e 1,225 fêmea/fêmea/dia $(\lambda)$ obtidos em algodoeiro em relação à T. pacificus e T. turkestani (Carey \& Bradley, 1982), respectivamente, sendo, porém, inferiores aos valores de 111,1, 0,290 fêmea/ fêmea/dia, 1,336 fêmea/fêmea/dia e 74,8, 0,219 fêmea/ fêmea/dia, 1,245 fêmea/fêmea/dia, constatados em algodoeiro em relação à T. desertorum (Nickel, 1960) e T. urticae (Carey \& Bradley, 1982). Em feijoeiro, Morros \& Aponte (1994) obtiveram, a $26^{\circ} \mathrm{C}$, os valores de 77,42 ovos, 19,63 dias, 0,25 e 1,287 fêmea/fê$\mathrm{mea} /$ dia, respectivamente, com relação à fecundidade, o tempo médio de uma geração, a razão intrínseca de crescimento, e a razão finita de crescimento de T. ludeni. Esses valores foram superiores aos obtidos no presente trabalho (Tabela 5). Desta forma, é provável que essas variações tenham ocorrido devido às diferenças entre substratos utilizados por cada um dos autores. Os valores da razão sexual obtidos nas cinco temperaturas foram semelhantes, e apresentaram maior proporção de fêmeas que de machos.

De modo geral, com o aumento da temperatura, houve tendência de aumento da fecundidade e diminuição do tempo médio de uma geração e, conseqüentemente, aumento de $\mathrm{r}_{\mathrm{m}} \mathrm{e} \mathrm{R}_{\mathrm{o}}$. Portanto, acreditase que T. ludeni tem grande potencial para causar danos econômicos nas regiões onde ocorrem temperaturas elevadas.

Tabela 5. Efeito de cinco temperaturas sobre os parâmetros biológicos de Tetranychus ludeni.

\begin{tabular}{lccccc}
\hline Parâmetro & \multicolumn{5}{c}{ Temperatura $\left({ }^{\circ} \mathrm{C}\right)$} \\
\cline { 2 - 6 } & 20 & 23 & 25 & 28 & 30 \\
\hline $\begin{array}{l}\text { Razão intrínseca } \\
\text { de crescimento }\left(\mathrm{r}_{\mathrm{m}}\right)^{(1)}\end{array}$ & 0,152 & 0,165 & 0,222 & 0,273 & 0,418 \\
$\begin{array}{l}\text { Razão finita } \\
\text { de aumento }(\lambda)\end{array}$ & 1,164 & 1,179 & 1,249 & 1,314 & 1,519 \\
$\begin{array}{l}\text { Razão de } \\
\text { crescimento }\left(\mathrm{R}_{\mathrm{o}}\right)\end{array}$ & 39,75 & 40,86 & 46,86 & 46,47 & 48,00 \\
$\begin{array}{l}\text { Tempo médio de } \\
\text { uma geração }(\mathrm{T})\end{array}$ & 24,20 & 22,47 & 17,30 & 14,07 & 9,27 \\
$\begin{array}{l}\text { Razão sexual } \\
\text { Fecundidade }\end{array}$ & 0,81 & 0,60 & 0,86 & 0,85 & 0,83 \\
$\begin{array}{l}\text { Número de } \\
\text { ovos/fêmea/dia }\end{array}$ & $43,55 \pm 18,65 \mathrm{bc}$ & $54,58 \pm 22,29 \mathrm{ab}$ & $51,13 \pm 27,26 \mathrm{ab}$ & $31,07 \pm 7,18 \mathrm{c}$ & $61,29 \pm 19,46 \mathrm{a}$ \\
\hline
\end{tabular}

${ }^{(1)}$ Fêmea/fêmea/dia. ${ }^{(2)}$ Dias. ${ }^{(3)}$ Fêmea/fêmea+macho. ${ }^{(4)}$ Médias seguidas pela mesma letra, na linha, não diferem entre si pelo teste de Tukey a $5 \%$ de probabilidade. 


\section{Conclusões}

1. Temperaturas entre $23^{\circ} \mathrm{C}$ e $30^{\circ} \mathrm{C}$ são favoráveis ao desenvolvimento de Tetranychus ludeni.

2. O efeito da temperatura no desenvolvimento de T. ludeni varia de acordo com a fase de desenvolvimento e o sexo.

3. Os valores das temperaturas-base e constantes térmicas variam conforme a fase de desenvolvimento, dentro de cada sexo e entre sexos de T. ludeni.

\section{Referências}

ALVAREZ, R. P. P.; ROJAS, L. A.; LOPEZ, E. de la T.; BARRIOS, M. D. Umbral mínimo de desarrollo de Tetranychus tumidus en el cultivo del plátano. Manejo Integrado de Plagas, Havana, n. 44, p. 26-28, 1997.

ANSARI, M. A.; PAWAR, A. D. Biology of spider mite, Tetranychus ludeni, Zacher (Acari: Tetranychidae) recorded on waterhyacinth. Plant Protection Bulletin, Faridabad, v. 44, n. 3, p. 28-31, 1992.

BIRCH, L. C. The intrinsic rate of natural increase of an insect population. Journal of Animal Ecology, Cambridge, Inglaterra, v. 17, p. 15-26, 1948.

BLEICHER, E. Importância relativa das principais pragas do algodoeiro em alguns estados do Brasil. Anais da Sociedade Entomológica do Brasil, Londrina, v. 22, n. 3, p. 554-562, 1993

BONATO, O. The effect of temperature on life history parameters of Tetranychus evansi (Acari: Tetranychidae) Experimental \& Applied Acarology, London, v. 23, n. 1, p. 11-19, 1999.

BOUDREAUX, H. B. Biological aspects of some phytophagous mites. Annual Review of Entomology, Palo Alto, v. 8, p. 137-154, 1963.

CALCAGNOLO, G. Influência do ataque do ácaro Eotetranychus telarius (L.) na produção algodoeira. Biológico, São Paulo, v. 29, p. 225-231, 1963.

CALCAGNOLO, G.; SAUER, H. F. G. Novos resultados no combate ao ácaro do algodoeiro Eotetranychus telarius (L.). Biológico, São Paulo, v. 21, p. 173-184, 1955.

CAREY, J. R.; BRADLEY, J. W. Developmental rates, vital schedules, sex ratios and life tables for Tetranychus urticae, T. turkestani and T. pacificus (Acarina: Tetranychidae) on cotton. Acarologia, Montpellier, v. 23, p. 333-345, 1982.
CARVALHO, J. P. de. Introdução à entomologia agrícola. Lisboa: Fundação Calouste Gulbenkian, 1986. 361 p.

JEPPSON, L. R.; KEIFER, H. H.; BAKER, E. W. Mites injurious to economic plants. Berkeley: University of California, 1975. $614 \mathrm{p}$.

KIM, S. S.; PAIK, C. H.; KIM, D. I.; PARK, J. D.; LEE, S. C. Some ecological characteristics of tea red spider mite, Tetranychus kanzawai Kishida (Acarina, Tetranychidae). Korean Journal of Entomology, Seoul, v. 23, n. 4 p. 261-266, 1993

KUMAR, V.; SHARMA, D. D. Bioecology and chemical control of spider mite, Tetranychus ludeni Zecher on okra Indian Journal of Plant Protection, Bihar, v. 21, n. 1, p. 68-71, 1993

MORAES, J. G. de; MacMURTRY, J. A. Effect of temperature and sperm supply on the reproductive potential of Tetranychus evansi (Acari: Tetranychidae). Experimental \& Applied Acarology, London, v. 3, p. $95-107,1987$.

MORROS, C. M. E.; APONTE, L. O. Biology and life Table of Tetranychus ludeni Zacher on black bean Phaseolus vulgaris L. Agronomia Tropical, Maracay, v. 44, n. 4 , p. $667-677,1994$

NAKANO, O.; SILVEIRA NETO, S.; ZUCCHI, R. A. Avaliação de danos e identificação prática das pragas: pragas do algodoeiro. In: Piracicaba: Esalq, 1981. p. 45-86

NICKEL, J. L. Temperature and humidity relationships of Tetranychus desertorum banks with special reference to distribution. Hilgardia, Berkeley, v. 30, n. 2, p. 41-100, 1960

POPOV, S. Y. On the identification of localities of spider mites (Acariformes, Tetranychidae) using biological characteristics. Zoologicheskii Zhurnal, Moscow, v. 73, n. $7 / 8$, p. $31-41,1994$

PRITCHARD, A. E.; BAKER, E. W. A revision of the spider mite family Tetranychidae. San Francisco: Pacific Coast Entomological Society, 1955. p. 405-406. (Memoirs Series, 2)

REDDY, G. V. P.; BASKARAN, P. Biology and varietal preference of Tetranychus ludeni Zacher (Acari: Tetranychidae) on four varieties of eggplant [aubergine], Solanum melongena L. Mysore Journal of Agricultural Sciences, Bangalore, v. 25, n. 3, p. 331-334, 1991

SINGH, R. N. Studies on seasonal abundance of Tetranychus ludeni Zacher (Acari: Tetranychidae) on 
cowpea (Vigna unguiculata Savi.). Journal of Recent Advances in Applied Sciences, Uttar Pradesh, v. 10, n. $1 / 2$, p. $59-63,1995$.

SUMANGALA, K.; HAQ, M. A. Microhabitat maintenance by Tetranychus ludeni (Acari: Tetranychidae).
Journal of Ecotoxicology \& Environmental Monitoring, Anna Nagar, v. 4, n. 3/4, p. 193-199, 1994

WILSON, L. J. Spider mites (Acari: Tetranychidae) affect yield and fiber quality of cotton. Journal of Economic Entomology, Lanham, v. 86, n. 2, p. 566-585, 1993. 